\title{
Verification of performance of Acuros XB Algorithm (AXB) Implemented on Eclipse Planning System
}

\author{
Nader Elsherbini ${ }^{1}$, Wafaa Ahmed ${ }^{2}$, Sherifa O. Elwakeel ${ }^{1}$
}

\author{
${ }^{1}$ Medical Physics Unit, Kasr Al-Ainy Center of Clinical Oncology and Nuclear Medicine, Kasr Al- \\ Ainy School of Medicine, Cairo University, Cairo, Egypt ; ${ }^{2}$ Department of Biophysics, Faculty of \\ Science, Cairo University, Cairo, Egypt.
}

Background: Verification of dose calculation algorithms is important in the radiotherapy process.
Aim: The aim of this work is to verify the dose calculation accuracy of Acuros XB version 10 algorithm (AXB10) in
homogenous and heterogeneous media. Methods: We compared AXB10 calculated doses with the measured doses using thermo-luminescent dosimeters (TLD) for $6 \mathrm{MV}$ photon beam. Two clinical cases, nasopharynx and lung tumors, were studied to evaluate the ability of the AXB10 to deal with tissue heterogeneity. Selected field sizes were measured in water phantom then compared with that calculated with the Eclipse treatment planning system (TPS) - based on AXB10 algorithm. Rando humanoid phantom was computerized tomography scanned and the images were transferred to the TPS system where a set of similar plans of a single direct field were calculated with AXB10, then the dose was measured within the phantom using the TLD in the brain and lung regions. Lastly the measured and calculated data were compared.

Results: There was a good agreement between the measured fields in the water phantom and that calculated with the AXB10 $( \pm 2 \%)$. The percentage difference between full Monte Carlo algorithm and AXB10 were 3\% for the phantoms with lung.

Conclusion: Acuros XB algorithm (version 10) results are in agreement with the International Commission for Units and Measurements recommendations.

Keywords: Acuros XB algorithm, Treatment planning system, Thermo-luminescent dosimeters Corresponding Author: Sherifa Elwakeel, Medical Physics Unit, Kasr Al-Ainy Center of Clinical Oncology and Nuclear Medicine, Kasr Al-Ainy School of Medicine, Cairo University, Cairo, Egypt, E-mail: sherifa_osman93@yahoo.com Submitted: 27-February-2018, Revised: 26-March-2018, Accepted: 28-March-2018, Published online: 8-April-2018

\section{INTRODUCTION}

Radiotherapy is a multi-step process starting with diagnosis and disease staging and ending with the treatment of specific target volume with predetermined radiation energies and beam parameters. The accuracy of the dose calculation and the dose delivery to the target has been increased especially in the last decades with the use of computed tomography (CT) which allowed more anatomical details and material information ${ }^{1}$. There are many steps to optimize the process of radiation therapy to deliver the given dose to the target in high accuracy ${ }^{2}$. The presence of heterogeneities causes dose calculation uncertainties which should be overcome to maximize the therapeutic benefit of radiation therapy ${ }^{2}$.

Acuros XB version 10 (AXB10) algorithm is based on Linear Boltzmann Transport Equation (LBTE) which is the linearized form of Boltzmann Transport Equation (BTE), the governing equation which describes the behavior of macroscopic particles (protons, neutrons, electrons, etc.) as they pass through and interact with the matter. Linear Boltzmann Transport Equation considers that the particle interacts only with the matter they are passing through not with the particles each other without the conditions of external magnetic field ${ }^{1}$.
Eclipse treatment planning system (TPS) is loaded by the AXB10 by Varian Medical Systems, Inc. (VMS) (Palo Alto, CA, USA). The origin of the AXB algorithm found in the Attila LBTE solver was developed by a group based on the founders of the Transpire, Inc. (Gig Harbor, WA, USA) ${ }^{2}$.

The accuracy of AXB11 was tested for 6 and 18 MV photon in a cubic water phantom which composed of stainless steel and titanium alloy. This resulted in a significant percentage of Gamma Analysis Index values of $96.8 \%$ and $91.3 \%$ for the comparison between the full Monte Carlo (MC) simulation and the AXB11 with acceptance criteria of $2 \%$ or $1 \mathrm{~mm}^{6}$.

Acuros XB version 10 and Analytical Anisotropic Algorithm (AAA) were tested for their accuracy in phantoms which composed of different percentages of solid-water and air, for different square and rectangular field sizes $\left(3 \times 3,5 \times 5,10 \times 10\right.$ and $\left.30 \times 30 \mathrm{~cm}^{2}\right)$ for $6 \mathrm{MV}$ photon beam. Phantom A composed of 2-cm air gap, phantom B composed of 4-cm air gap, and phantom C composed of $6-\mathrm{cm}$ air gap. The three phantoms were imaged by computed tomography (CT) and transferred to the Eclipse Planning System via DICOM for calculation by AXB10 then recalculated by AAA. The deviation between AXB10 and measured data was from 
$-3.81 \%$ to $+0.9 \%$, while the deviation between AAA and measured data was from $-3.1 \%$ to $-10.9 \%{ }^{7}$.

In a study that included lung cancer cases presenting for radiotherapy, the planning target volume (PTV) was calculated by AXB11 and recalculated by AAA. The mean deviation was less than $2 \%$ for the PTV and less than $3 \%$ for the organs at risk ${ }^{9}$. While the cases treated by stereotactic body radiation therapy technique needed more monitor units (MU) due to the presence of heterogeneities ${ }^{10}$.

In breast cancer cases receiving conformal treatment in the form of two tangential fields of $6 \mathrm{MV}$, AXB predicted lower doses to the PTV and to the lung compared to AAA. So the AXB may be needed for improving low density materials' calculations ${ }^{11}$.

The aim of this study was to test the accuracy of AXB10 in heterogeneous (nasopharynx and lung) and homogenous (water phantom) regions.

\section{METHODS}

\section{Materials}

Linear Accelerator: The unique Linac (UNIQUE TM, Varian Palo Alto, California, USA) was used in this study with field size ranging from $4 \times 4$ to $40 \times 40$ $\mathrm{cm}^{2}$ at a $100 \mathrm{~cm}$ Source to Skin Distance (SSD). The linear accelerator was calibrated so that a square symmetric field $\left(10 \times 10 \mathrm{~cm}^{2}\right)$ will deliver an absorbed dose of $1 \mathrm{~Gy}$ per $100 \mathrm{MU}$ at $10 \mathrm{~cm}$ depth in water with an SSD of $90 \mathrm{~cm}$. Gantry Rotation angle from the vertical was $\pm 185^{\circ}$. Mechanical and Radiation Isocenter Accuracy was $<0.1 \mathrm{~cm}$ radius sphere. For arc technique, the gantry was not to deviate $>7$ degrees from the desired angle which was considered as 0.2 MU from the total dose.

Treatment planning system (TPS): The Eclipse TM Treatment Planning Software (version 10.0) with AXB10 and AAA with the same version used in this study, is an integrated and comprehensive system supporting radiation treatment (e.g. photons, flattening filter free beams, protons, electrons, external beams, low-dose-rate brachytherapy and cobalt therapy).

Water Phantom: PTW (Freiburg, MP1) water tank is remote controlled and has dimensions of 254 $\mathrm{mm} \times 320 \mathrm{~mm} \times 370 \mathrm{~mm}$. It is enhanced by holding devices and TBA electronics and MEPHYSTO $\mathrm{mc}^{2}$ software for software operations.

Humanoid Rando: Alderson RANDO phantom (ART) (Radiology Support Devices Inc., Long Beach, CA) was used in this study. The phantom is $175 \mathrm{~cm}$ tall and $73.5 \mathrm{~kg}$ weight. It consists of 34 slices each of 2.5 $\mathrm{cm}$ thickness. Each slice is perforated for the insertion of thermo-luminescent dosimeter (TLD) chips. The phantom is made of tissue-equivalent material according to International Commission on Radiation Units and Measurements (ICRU) - 44 recommendations. The skeleton is composed of polymer moldings to simulate the shape, mass, density and the lung is molded from synthetic foam.

Computed Tomography (CT): The multislice CT machine used in this work was General Electric (UK); light speed RT. This CT machine can record $4 \mathrm{CT}$ images per rotation and the CT unit is provided with a flat carbon fiber table to simulate the Linac couch.

Thermo-Luminescent Dosimeter (TLD): System $\mathrm{LiF}$ (700) chips were used in this study for measuring dose in the humanoid. The chip material was $\mathrm{LiF}$ enriched with $\mathrm{Mg}$ and $\mathrm{Ti}$ impurities (each chip is 0.45 $\mathrm{cm}$ [diameter] x $0.08 \mathrm{~cm}$ [thickness]) with an effective atomic number of 7.4. The system also included a Rados TLD reader unit which was used to read the dose measured by TLD chips.

\section{Procedures}

Verification of AXB10 depth dose and beam profile measurements: Phantom of $40 \times 40 \times 40 \mathrm{~cm}^{3}$ was created as a 3D structure on Eclipse planning system, which was defined as body structure (CT value $=0$ ) and used for computation of percentage depth doses (PDDs) and beam profiles for calculated field sizes for AXB10. This was then compared to the measured data with the water phantom at $S S D=100 \mathrm{~cm}$ for $6 \mathrm{MV}$ photon beam. The PDDs were normalized to $100 \%$. Beam profiles were measured and calculated at depths of $1.5,5$ and $15 \mathrm{~cm}$ for the $6 \mathrm{MV}$ photon beam.

TLD (LiF 700) preparation procedures: A new batch of LiF (700) chips was used. The chips were annealed before use by heating up to $400^{\circ} \mathrm{C}$ for one hour and for two hours at $100^{\circ} \mathrm{C}$ then left in the oven to cool to room temperature. All chips were then irradiated to $1 \mathrm{mGy}$ using RADOS irradiator. Then sensitivity factor for each TLD (LiF 700) chip was measured by dividing the TLD ( $\mathrm{LiF} 700)$ reader count by the given dose. The average factor was calculated for all chips and considered as $100 \%$. All chips with sensitivity factors exceeding $\pm 2 \%$ were omitted. The absorbed doses were measured by TLD in two CT cuts in RANDO phantom in nasopharnyx and lung regions to test AXB10 in the heterogeneity regions in soft tissue , air -bone interface and air-soft tissue interface.

Evaluation of AXB10: One of the error sources in dose calculations arises from the ability of TPS to deal with tissue heterogeneity. Humanoid RANDO phantom was CT scanned with slice spacing of 2.5 $\mathrm{mm}$. These series were transferred to the TPS through DICOM network. Organs at risk were delineated in the phantom; including right lung, left lung, heart and spinal cord in the chest region and right optic nerve, left optic nerve, brain stem and optic chiasm in the brain region. A plan of single isocentric direct field size of $20 \times 20 \mathrm{~cm}^{2}$ was created and doses at certain points (representing the positions of TLD chips) were calculated and recorded. Plan isocenter position was verified with the simulation step in which the anterior and lateral Digital Reconstructed Radiography views were compared to the corresponding 2D simulator images. The TLD chips were inserted in their predefined positions in which two crystals were inserted in each position and irradiated with the previously mentioned field .Then crystals were collected and the dose was measured by the TLD reader. The previous step was repeated 3 times for accuracy.

Dose assessment: 1) Two plans were created in the TPS for nasopharynx and lung cases and then 
calculated by AXB10, 2) The measured and calculated doses were compared. For TLD measurements the percentage difference between measured and calculated doses was calculated as follows:

$\%$ Dose difference $=100 \times(($ Dose measured Dose calculated) / Dose measured)

\section{RESULTS}

\section{Measurements of tissue heterogeneity}

In the lung case, the differences between measured and calculated dose in the heterogeneous regions ranged from -0.7 to 0.4 (table 1 ). In the nasopharynx case the differences ranged from 0.04 to 0.1 (table 2).

\section{Comparison between standard measurements}

The PDDs of field sizes $5 \times 5,10 \times 10,15 \times 15$ and $25 \times 25 \mathrm{~cm}^{2}$ were compared between the measured PDDs from the water phantom and the calculated PDDs from the TPS loaded by AXB10 and showed standard deviations of $0.3 \%, 0.2 \%, 0.1 \%$ and $0.1 \%$ respectively (figure 1).

The standard deviations for the dose profiles of field sizes $5 \times 5,10 \times 10,15 \times 15$ and $25 \times 25 \mathrm{~cm}^{2}$ between measured and calculated doses in the three depths $1.5,5$, and $15 \mathrm{~cm}$ were in the range of $0.1-0.3,0.2-0.3$ and 0.1 0.4 respectively (figure 2 ).

Table 1. The TLD measured doses and the Eclipse TPS calculated relative doses in the lung region

\begin{tabular}{llllllll}
\hline Energy & Point No. & Region & TLD & Eclipse (Acuros XB 10 Algorithm) & Diff.(\%) & Met criteria \\
\hline 6MV & 656 & Bone air interface & 117.2 & 118 & -0.7 & Yes & \\
\hline 6MV & 655 & Air soft tissue interface & 192.5 & 193.1 & -0.6 & Yes \\
\hline $6 \mathrm{MV}$ & 657 & Soft lung & 157.4 & 157 & 0.4 & Yes
\end{tabular}

TLD: Thermo-luminescent dosimeters

Table 2. The TLD measured doses and the Eclipse TPS calculated relative doses in the Nasopharynx region

\begin{tabular}{llllllll}
\hline Energy & Point No. & Region & TLD & Eclipse (Acuros XB 10 Algorithm) & Diff.(\%) & Met criteria \\
\hline 6MV & 798 & Soft tissue & 1.3 & 1.4 & 0.1 & Yes \\
\hline 6MV & 797 & Bone region & 0.99 & 1.1 & 0.2 & Yes \\
\hline $6 \mathrm{MV}$ & 796 & Tissue-Air interface & 1.76 & 1.8 & 0.04 & Yes \\
\hline
\end{tabular}

TLD: Thermo-luminescent dosimeters

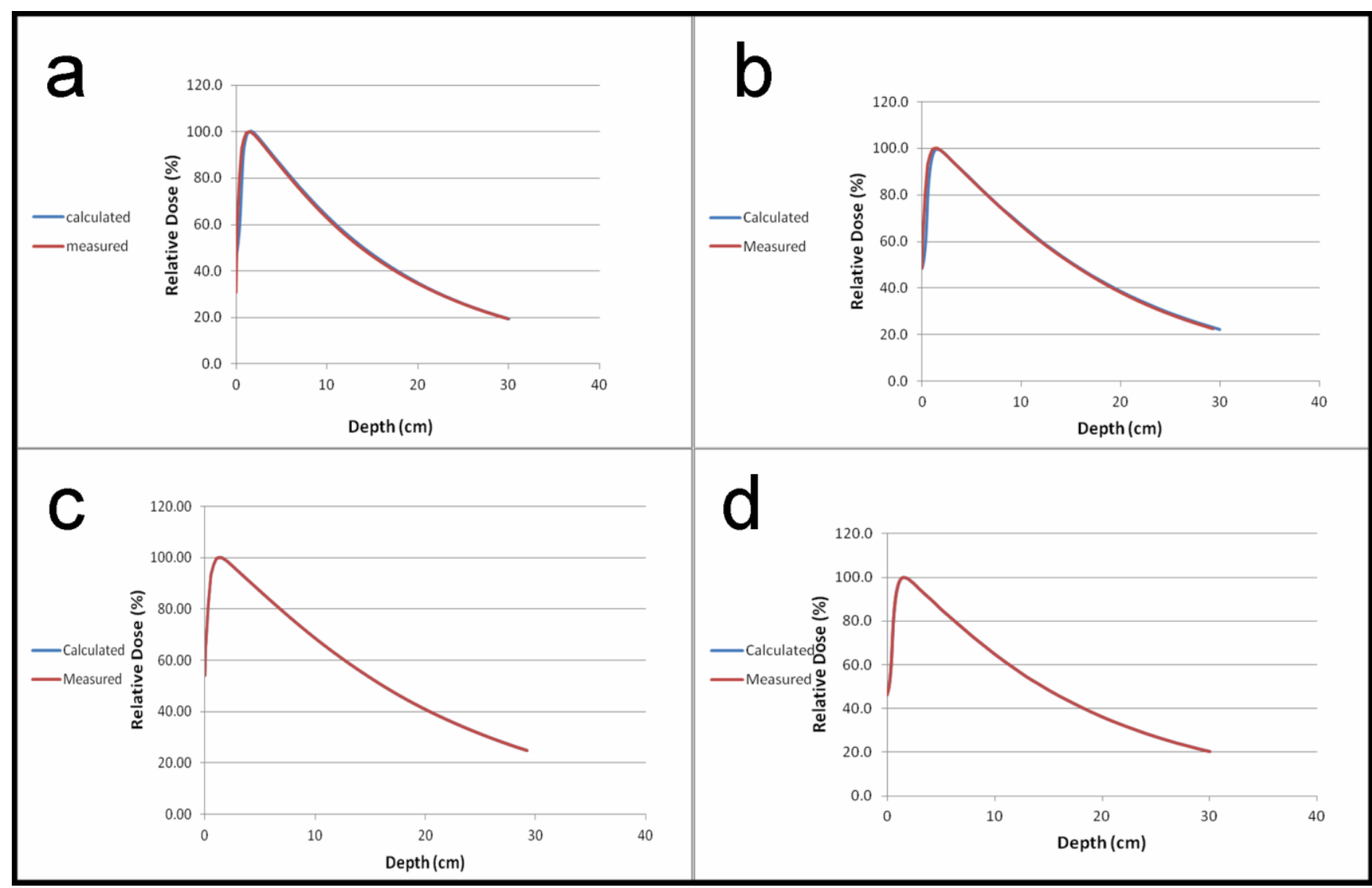

Figure 1. Measured and calculated percentage depth dose for field sizes: a) $5 \times 5 \mathrm{~cm}^{2}$ at SSD $=100 \mathrm{~cm}$, b) $10 \times 10 \mathrm{~cm}^{2}$ at $\mathrm{SSD}=100 \mathrm{~cm}, \mathbf{c}) 15 \times 15 \mathrm{~cm}^{2}$ at $\mathrm{SSD}=100 \mathrm{~cm}$ and d) $25 \times 25 \mathrm{~cm}^{2}$ at $\mathrm{SSD}=100 \mathrm{~cm}$ 


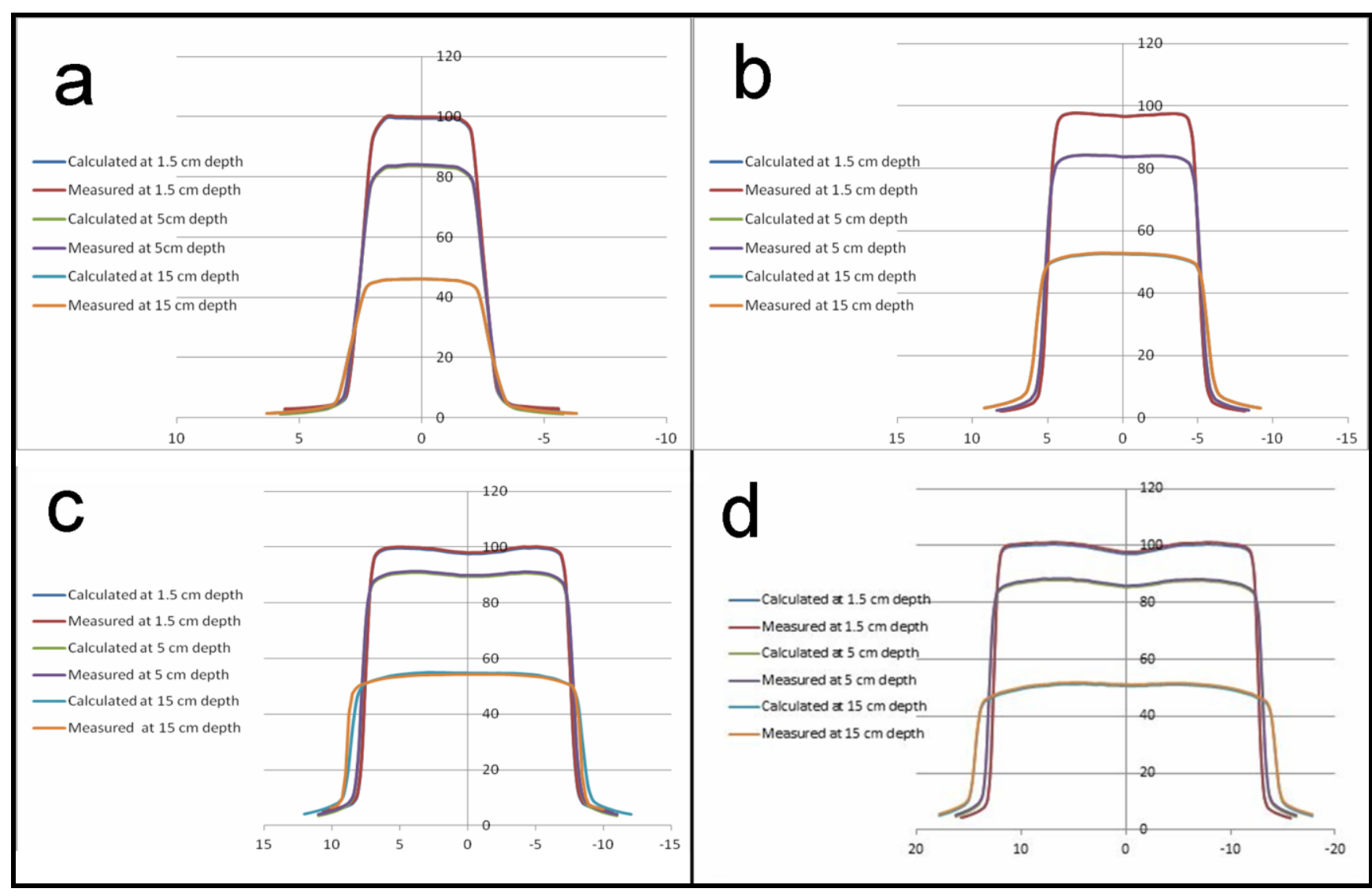

Figure 2. Measured and calculated profiles for field sizes: a) $5 \times 5 \mathrm{~cm}^{2}$ at SSD $=100 \mathrm{~cm}$, b) $10 \times 10 \mathrm{~cm}^{2}$ at $\mathrm{SSD}=100 \mathrm{~cm}$, c) $15 \times 15 \mathrm{~cm}^{2}$ at $\mathrm{SSD}=100 \mathrm{~cm}$ and d) $25 \times 25 \mathrm{~cm}^{2}$ at $\mathrm{SSD}=100 \mathrm{~cm}$

\section{DISCUSSION}

Tissue heterogeneity could be a source of error which leads to errors in radiotherapy dose delivery resulting from TPS. This highlights the importance of TLD testing to verify the accuracy of algorithms.

The American Association of Physicists in Medicine (AAPM) recommended the proportion of each source of uncertainties, determined for each step in the radiotherapy process for achieving the accuracy, to be in the range from $1.0 \%$ to $5.0 \%$. This was decreased below $3.0 \%$ by the Report 85 of Task Group 65 of AAPM ${ }^{3}$. Our results for homogeneity are consistent with these recommendations.

Also, ICRU report no. 85 has recommended that the accuracy of the absorbed dose to be $5 \%$ and the AAPM has recommended that the uncertainty in computed dose distribution to be less than $2 \%{ }^{4}$. According to ICRU report no. 83, the deviation on the delivered dose to the PTV should be within $+3 \%$ or $-3 \%{ }^{5}$. The results of the current study for heterogeneity are congruent with these recommendations.

Our results are also in good agreement with other studies. Bush et al reported that the percentage difference between the AXB10 and full MC simulations was within $3 \%$ for the phantoms with lung inserted ${ }^{11}$. Also, Fogliata et al tested AXB10 and AXB11 in water phantom for square field sizes ranging from $2 \times 2$ to $40 \times 40 \mathrm{~cm}^{2}$ using flattening filter free $6 \mathrm{MV}$ beam. They found that the percentage difference was within $1 \%$ for open beams ${ }^{12}$.

\section{Conclusion}

In this study we tested the accuracy of AXB10 in water phantom with the same calculations from TPS which gave a good agreement.

The results of the work showed that AXB10 made an improvement in beam modeling in homogenous medium (water phantom) measurement for both PDDs and beam profiles measurement. Also measurements in heterogeneous medium (RANDO phantom) showed good results between measured doses in the lung region by both TPS loaded by AXB10 and doses delivered by LINAC.

\section{Acknowledgement}

The authors would like to express their gratitude to Physicist. Mohamed Sayed, MSc (Kasr Al-Ainy Center of Clinical Oncology and Nuclear Medicine, Cairo University) for his valuable support.

\section{Conflict of interest}

None to declare

\section{REFERENCES}

1. Ojala $J$. The accuracy of the Acuros XB algorithm in external beam radiotherapy - a comprehensive review. Int J Cancer Ther Oncol. 2014; 2(4): 020417. 
2. Vassiliev ON, Wareing TA, Davis IM, et al. Feasibility of a multigroup deterministic solution method for threedimensional radiotherapy dose calculations. Int $\mathbf{J}$ Radiat Oncol Biol Phys. 2008; 72(1):220-227.

3. Papanikolaou N, Battista JJ, Boyer AL, et al. Report of Task Group No. 65 of the Radiation Therapy Committee of the American Association of Physicists in Medicine: Tissue inhomogeneity corrections for megavoltage photon beams. Madison, WI: Medical Physics Publishing, 2004

4. International Commission on Radiation Units and Measurements. Report 85: Fundamental quantities and units for ionizing radiation. J ICRU. 2011; 11(1): 1-31.

5. International Commission on Radiation Units and Measurements. Report 83: Prescribing, Recording, and Reporting Photon-Beam Intensity-Modulated Radiation Therapy (IMRT). J ICRU. 2010; 10(1): 1-106.

6. Lloyd SA, Ansbacher W. Evaluation of an analytic linear Boltzmann transport equation solver for high-density inhomogeneities. Med Phys. 2013; 40(1): 011707.

7. Liu HW, Nugent Z, Clayton R, Dunscombe P, Lau H, Khan R. Clinical impact of using the deterministic patient dose calculation algorithm Acuros $\mathrm{XB}$ for lung stereotactic body radiation therapy, Acta Oncol. 2014; 53: 324-329.
8. Rana S, Rogers K, Pokharel S, Cheng C. Evaluation of Acuros XB algorithm based on RTOG 0813 dosimetric criteria for SBRT lung treatment with Rapid Arc. J Appl Clin Med Phys. 2014; 15(1): 4474.

9. Rana S, Rogers K, Lee T, Reed D, Biggs C. Verification and dosimetric impact of Acuros XB algorithm for stereotactic body radiation therapy (SBRT) and RapidArc planning for non-small-cell lung cancer (NSCLC) patients. Int J Med Phys Clin Eng Radiat Oncol. 2013; 2: 6-14.

10. Warren S, Panettieri V, Panakis N, et al. Optimizing collimator margins for isotoxically dose-escalated conformal radiation therapy of non-small cell lung cancer. Int J Radiat Oncol Biol Phys. 2014; 88(5): 1148-1153.

11. Bush K, Gagne IM, Zavgorodni S, Ansbacher W, Beckham W. Dosimetric validation of Acuros XB with Monte Carlo methods for photon dose calculations. Med Phys. 2011; 38(4): 2208-2221.

12. Fogliata A, Nicolini G, Clivio A, Vanetti E, Cozzi L. Accuracy of Acuros XB and AAA dose calculation for small fields with reference to RapidArc(®) stereotactic treatments. Med Phys. 2011; 38(11): 6228-6237. 